OPEN ACCESS

Edited by:

Thomas Tessonnier, Heidelberg University Hospital,

Germany

Reviewed by:

Tatsuya Ohno,

Gunma University, Japan

Thomas Held,

Heidelberg University Hospital,

Germany

*Correspondence:

Barbara Vischion

barbara.vischioni@cnao.it

Specialty section:

This article was submitted to

Radiation Oncology,

a section of the journal

Frontiers in Oncology

Received: 05 December 2021

Accepted: 04 February 2022

Published: 02 March 2022

Citation:

Vischioni B, Russo S, Meuli M,

Bonora $M$, Ronchi S, Ingargiola $R$, Camarda AM, Imparato S, Preda L, Ciocca M, Molinelli S and Orlandi E

(2022) Dosimetric and Clinical

Risk Factors for the Development of Maxillary Osteoradionecrosis

in Adenoid Cystic Carcinoma

(ACC) Patients Treated With

Carbon Ion Radiotherapy.

Front. Oncol. 12:829502.

doi: 10.3389/fonc.2022.829502

\section{Dosimetric and Clinical Risk Factors for the Development of Maxillary Osteoradionecrosis in Adenoid Cystic Carcinoma (ACC) Patients Treated With Carbon Ion Radiotherapy}

\author{
Barbara Vischioni ${ }^{1 *}$, Stefania Russo ${ }^{1}$, Martino Meuli ${ }^{2}$, Maria Bonora ${ }^{1}$, Sara Ronchi ${ }^{1}$, \\ Rossana Ingargiola ${ }^{1}$, Anna Maria Camarda ${ }^{3,4}$, Sara Imparato ${ }^{1}$, Lorenzo Preda ${ }^{1,2,5}$, \\ Mario Ciocca ${ }^{1}$, Silvia Molinelli ${ }^{1}$ and Ester Orlandi ${ }^{1}$

\begin{abstract}
${ }^{1}$ Radiation Oncology Clinical Department, National Center for Oncological Hadrontherapy, Pavia, Italy, ${ }^{2}$ Department of Clinical, Surgical, Diagnostic and Pediatric Sciences, University of Pavia, Pavia, Italy, ${ }^{3}$ Division of Radiotherapy, Istituto Europeo di Oncologia (IEO) European Institute of Oncology, Istituto di Ricovero e Cura a Carattere Scientifico (IRCCS), Milan, Italy, ${ }^{4}$ Department of Oncology and Hemato-Oncology, University of Milan, Milan, Italy, ${ }^{5}$ Radiology Institute, Fondazione Istituto di Ricovero e Cura a Carattere Scientifico (IRCCS) Policlinico San Matteo, Pavia, Italy
\end{abstract}

Background: The present study aims to evaluate dosimetric and clinical risk factors for the development of maxillary osteoradionecrosis (ORN) in head and neck adenoid cystic carcinoma (ACC) patients treated with carbon ion radiotherapy (CIRT).

Methods: Clinical data and treatment plans of ACC patients, consecutively treated from January 2013 to September 2016 within the phase II clinical trial CNAO S9/2012/C, were retrospectively reviewed. ORN and other treatment-related toxicity were graded according to the Common Terminology Criteria for Adverse Events (CTACE), version 4.0. The maxillary bone was contoured on the planning CT, and only patients receiving more than $10 \%$ of the prescription dose at their maxilla were considered for the analysis (67 patients). The volumes of maxilla receiving doses from 10 Gy (RBE) to 60 Gy (RBE) $\left(V_{D}\right)$, with an increment of $10 \mathrm{~Gy}(\mathrm{RBE})$, and additional clinical factors were correlated to the incidence of ORN with univariate analysis (Chi-square test). The logistic regression model was subsequently applied for multivariate analysis. Treatment plans calculated with a local effect model (LEM)-based optimization were recalculated with the modified microdosimetric kinetic model (MKM), and compared with literature data from the Japanese experience.

Results: The median time interval from the start of CIRT to ORN appearance was 24 months (range, 8-54 months). Maxillary ORN was observed in 11 patients (16.4\%). Grade 1 ORN was observed in 2 patients (18.1\%), G2 in 4 (36.3\%), G3 in 4 (36.3\%) and G4 in 1 (9.3\%). From univariate analysis, the site of the tumor, the presence of teeth within the PTV and acute mucositis correlated with the development of maxillary ORN. $V_{D}$ were significantly higher for all the dose levels tested in patients with maxillary ORN than 
patients without necrosis, according to both radiobiological models. The multivariate analysis showed that V60 significantly correlated with ORN risk.

Conclusion: The volume of maxilla irradiated with high dose values was relevant for ORN development in our cohort of ACC patients. These results are in line with previously published data obtained with a different radiobiological model. Our findings might be helpful to prevent the risk of ORN in patients receiving CIRT.

Keywords: ACC, maxillary osteonecrosis, carbon ion radiotherapy, risk factors, late toxicity

\section{INTRODUCTION}

Osteoradionecrosis (ORN) is described as a chronic wound that fails to heal with bone exposure. It is a treatment related complication primarily reported in the head and neck population treated with radiotherapy (RT) frequently associated with a substantial morbidity causing pain and infection. The pathophysiology of its occurrence is linked to the hypoxia, hypocellularity, and hypo vascularity that follow a RT course (1). There are several risk factors that have been reported to favor ORN in patients undergoing RT, for example alcohol and tobacco consumption, dental and periodontal status deterioration, trauma after dental extractions, oral implantology, insufficient body mass index, general comorbidities such as diabetes, and anti-inflammatory or anti-coagulant therapy (2). Treatment related factors that might be linked to ORN are technique of irradiation, dosimetric parameters, fractionation schedule, extension of irradiated volumes, and concomitant chemotherapy (3).

The incidence of ORN of the mandible when irradiating parotid tumors and also at the skull base when irradiating tumors localized at the nasopharynx, has decreased in recent years with the transition from 2 and 3D-RT to the modern intensity modulated-RT (IMRT), since the more localized deposition of the higher doses to the tumor target in respect to the surrounding normal tissues $(4,5)$. In this regard, carbon ion RT (CIRT) has shown its superiority in dosimetric studies in delivering tumoricidal dose while sparing the surrounding normal tissues, due to the sharp penumbra of the therapeutic beams. Furthermore, the radiobiological properties of CIRT that causes not repairable DNA damage not cell cycle specific nor oxygen dependent (6), have advocated its therapeutic use for radioresistant tumors, such as adenoid cystic carcinoma (ACC). The gold standard treatment for ACC is radical surgical resection followed by post-operative RT (7). In case of unresectable locally advanced tumors or with tumor macroscopic persistence after surgical resection or patient contraindication to surgery $(8-10)$, or reirradiation (11), CIRT has been recently shown to have higher efficacy compared to conventional photon RT (12).

While numerous clinical and physical factors have been reported to be associated with ORN development after photon $\mathrm{RT}$, the risk factors for maxillary ORN after CIRT are still under investigation. Sasahara et al. (13) reported V50 as a good predictor of ORN in multivariate analysis in a series of 63 malignant radioresistant tumors (namely, 24 ACC) treated with CIRT at a prescribed dose of $57.6 \mathrm{~Gy}$ (RBE) in 16 fractions, with passive beam delivery. RBE-weighted dose calculation was based on the semi-empirical model by Kanai et al. (14), while the modified microdosimetric kinetic model (MKM) is currently used for pencil beam scanning in Japan (15). The two models have been validated for consistency, and only the latter will be adopted here for comparison against available literature data.

The aim of the present study was to identify dosimetric and non-dosimetric risk factors correlated to ORN development for head and neck ACC patients treated at CNAO (National Center for Oncological Hadrontherapy, Pavia, Italy) with CIRT delivered with curative intent. At CNAO the local effect model (LEM-version I) is employed for RBE calculation as in other European CIRT centers (16), with treatment protocols derived from the Japanese experience (17), after mice and cell experiments to assure comparable radiobiological efficacy of the CNAO carbon ion beam to the reference CIRT beam of the NIRS Hadrontherapy Center in Japan (18). The impact of RBE conversion from MKM to LEM on prescription doses and organ at risk (OAR) constraints was extensively reported for different CIRT cancer indications, including ACC (19). Only MKM model-based analysis on ORN has been reported so far for patients with head and neck tumors (13), while here we directly investigate risk factors for ORN development when irradiating head and neck patients with CIRT by using the LEM model. The findings from the present study might be helpful to prevent ORN risk at the maxilla in patients receiving CIRT in facilities where the LEM is used for treatment of patients in 16 fractions, directly estimating the dose that might be delivered without causing ORN, and thus avoiding the deterioration of quality of life reported for example when ORN evolves into oronasal fistula (20). Furthermore, in our study the identification of other clinical and treatment related risk factors predisposing to ORN might be helpful to assess ORN risk in the head and neck population undergoing CIRT and plan adequate follow up schedule with possible preventive measures.

\section{MATERIAL AND METHODS}

\section{Study Protocol Design}

The present study is a retrospective, observational study, a subanalysis of the prospective phase II clinical trial ongoing 
from January 2013 to September 2016 at CNAO (CNAO S9/ 2012/C) to assess safety and feasibility of CIRT with radical intent for ACC patients with partially resected tumors (R1/R2 margins), or unresectable tumors, or unfit to surgery (21). CNAO's Ethics Committee formally approved the present research protocol CNAO OSS/19/2020, and the patient consents for the research participation were properly collected and stored. The primary endpoint of the present study was to evaluate the dose of CIRT associated with the development of ORN at the maxillary bone in the ACC patients treated at CNAO with CIRT according to the LEM model. Secondary endpoints were the correlation of ORN in our series with the patient clinical and treatment characteristics to describe possible factors that might predict bone toxicity after CIRT.

\section{Patient's Baseline and Follow-Up Data}

A total of 128 patients with head and neck ACC consecutively treated with CIRT along with their diagnostic imaging and treatment plan data were screened for selection in the present study. Only the patients with at least more than $10 \%$ of the total prescription dose of $68.8 \mathrm{~Gy}$ (RBE) to their maxilla were selected for the analysis as detailed in Sasahara et al. (13). On the other side, patients with tumor invading the maxillary bone before CIRT, or with maxillary recurrence after CIRT, were excluded to avoid confounding factors for ORN assessment. At the end, 67 patients, 35 women $(52.2 \%)$ and 32 men $(47.8 \%)$ were considered for the analysis. The mean age of the studied population at the beginning of the CIRT course was of 55 years. Patients clinical and tumor characteristics (namely, anamnestic data that have been reported to impact on ORN except for osteoporosis), and data related to previous surgery and CIRT toxicity, were collected and detailed in Table 1. Most of the tumors were in the sinonasal area (35.8\%). The other sites involved were the parotid glands (20.9\%), the pharynx (17.9\%), the submandibular glands (13.4\%), the oral cavity $(9 \%)$, and the external auditory channel (3\%). Tumors were all re-staged following the 8th edition of TNM staging system (AJCC/UICC). Prior to therapy, the patients were adequately informed about the possible risks of maxillary ORN as

TABLE 1 | Patients clinical and tumor characteristics.

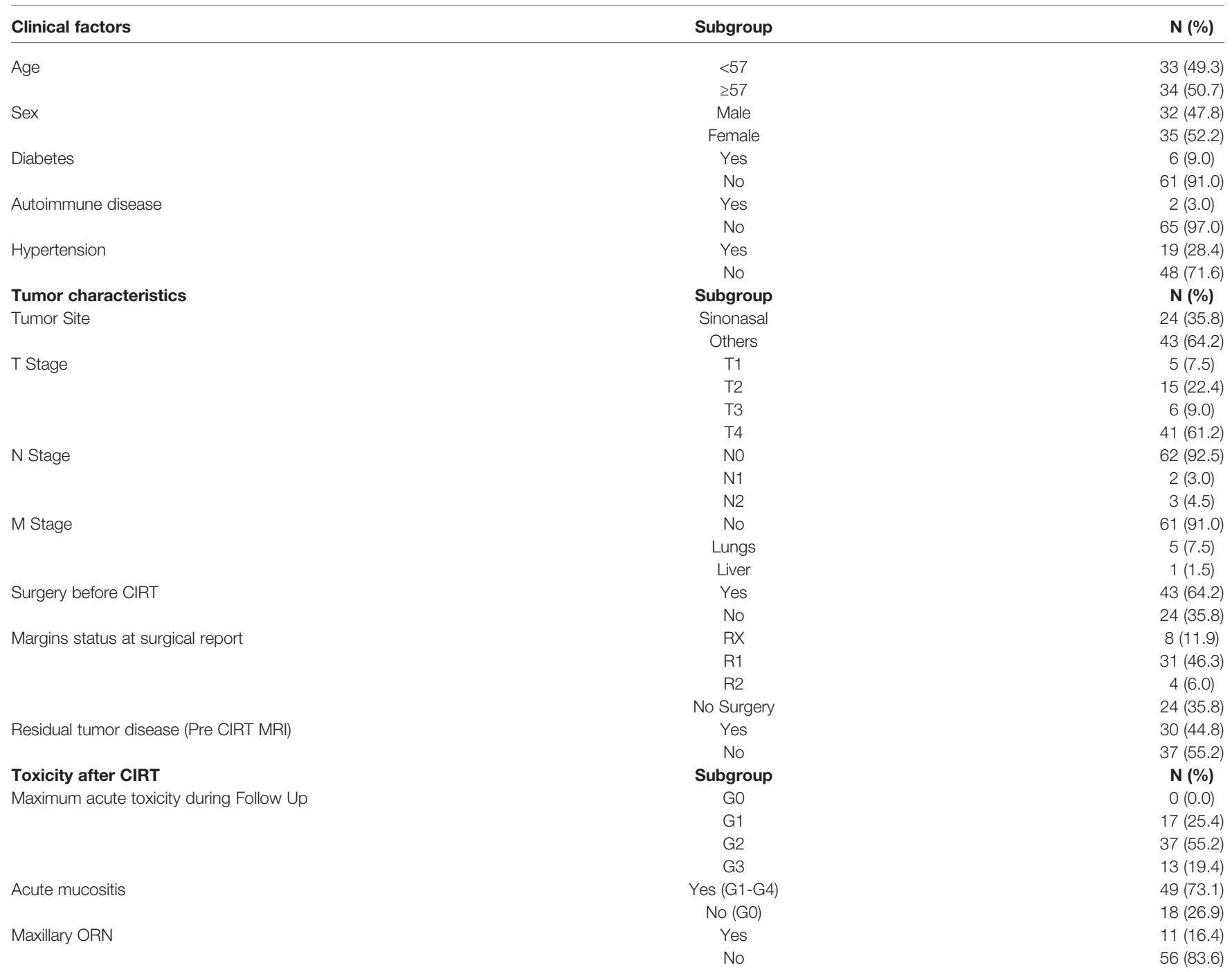


a side effect after irradiation. As of institutional practice, a complete dental examination and a dental-periodontal prophylaxis were requested. If indicated, atraumatic extraction of dental elements with a poor prognosis was performed at least 10 days prior to treatment. For each patient, a dental health certificate stating the good dental health was mandatory to start CIRT. No patient underwent bisphosphonates or concomitant systemic treatment during CIRT or adjuvant chemotherapy after CIRT. After CIRT patients were regularly followed up every 3 months for at least 2 years, then every 6 months since the fifth year, with magnetic resonance imaging (MRI) examination and fibroscopic local examination, with a staging total body Computed Tomography (CT) once per year. The diagnosis of maxillary ORN relied on the clinical symptoms reported by each patient, the oral-maxillo-facial physical examination, and the imaging scan acquired during the follow up reviewed by a senior radiologist. In case of maxillary ORN a maxillo-facial CT scan was also performed along with the follow up MRI to further study the affected bone. Acute and late toxicities were collected at each follow up clinical visit and scored according to the Common Terminological Criteria for Adverse Events (CTCAE), version 4 (22), including bone toxicity.

\section{Carbon Ion RT Treatment Planning and Dose-Volume Histogram Analysis}

A set of $2 \mathrm{~mm}$ thickness CT scan was acquired for treatment planning. Patients were immobilized with a personalized thermoplastic head-neck-shoulder mask equipped with biteblocks and fixation points to the couch as previously reported (23). After the simulation CT, a MRI was acquired in the same setup condition. The gross tumor volume (GTV) was first outlined based on the morphological analysis of the planning CT after registration with MRI, namely, the primary tumor lesion or the surgical tumor bed, and the metastatic lymph nodes. The high-risk clinical target volume 1 (CTV1) was delineated by including the GTV and a minimum of $5 \mathrm{~mm}$ margin in all directions to cover the microscopic extent of the lesion. A larger CTV2 (including the CTV1) with the anatomical area at risk of perineural spread along the cranial nerves up to the skull base was also outlined. Irradiation to prophylactic lymph nodes was omitted. PTV1 and PTV2 were created, adding a 2 $\mathrm{mm}$ margin at each CTV. The total prescription dose was 68.8 Gy (RBE) in 16 fractions over 4 weeks (4.3 Gy (RBE)/fraction, 4 fractions/week). CTV2 was irradiated with a dose of $38.7 \mathrm{~Gy}$ (RBE) and the high-risk CTV1 was irradiated up to the total prescribed dose. Patients were treated with 2 to 4 fields with active pencil beam scanning technique. Treatment plans were optimized with Syngo PT (Siemens AG Healthcare, Erlangen, Germany) treatment planning system (TPS), and the LEM I model was used for RBE-weighted dose calculation with an ideal $\alpha / \beta$ ratio of $2 \mathrm{~Gy}$. For this study, all treatment plans were recalculated with a research version of Raystation TPS (V6.99, Raysearch, Stockholm, Sweden), which provides both LEM I and MKM for RBE-weighted dose calculation.

The radiological morphology of the maxillary bone was first studied and then manually contoured on planning CT for each patient. The delineation of maxillary volumes included the alveolar process, the palatine process and the roots of the upper teeth in their own alveoli as previously reported (13). Pterygoid processes, sinus cavities and the crowns of the upper teeth were excluded. The mean maxillary volume in the patient series was $26.2 \mathrm{ml}$ (range: 11.1-42 ml). The absolute maxillary volumes receiving doses $\mathrm{D}$ ranging from 10 to $60 \mathrm{~Gy}(\mathrm{RBE})$ were extracted from the dose volume histograms (DVHs) of the patients, and were expressed as $\mathrm{V}_{\mathrm{D}}$ doses (V10, V20, V30, V40, V50, and V60), for both LEM and MKM calculated plans. For each $V_{D}$ parameter of the LEM-based plans, the median value was calculated over the entire patient cohort, and patients dichotomized accordingly, to test the correlation with the incidence of ORN. Furthermore, the patient cohort was divided into two groups according to the appearance of ORN, and the average DVHs were calculated for both groups and radiobiological models (LEM and MKM). In the work by Sasahara et al., the absolute maxillary volume receiving $50 \mathrm{~Gy}$ (RBE) (V50 $0_{\mathrm{MKM}}$ ) was found significant for the onset of ORN. We therefore extracted the V50 ${ }_{\mathrm{MKM}}$ for each patient, from the MKM-DVH, and calculated the corresponding LEM-based dose received by the same volume of maxilla in each delivered plan, to compare our treated population with literature dosimetric data (13). For each LEMbased patient treatment plan, the presence of teeth in the irradiated PTV was recorded for analysis.

\section{Statistical Analysis}

The Chi-square test was performed to investigate both clinical and dosimetric variables as predictors of ORN risk. For statistically significant variables only, the strength of association was estimated by Cramer's V. The Kaplan-Meier method was adopted to evaluate the cumulative incidence of late post-radiation bone effects with respect to the only statistically significant variables in the univariate analysis. The difference that was observed between the cumulative incidence curves was compared with the Log-rank test. P-values $<0.05$ were considered statistically significant. The logistic regression model was subsequently applied to assess how the baseline risk of developing ORN varied according to the presence/absence of several given variables, namely, the $\mathrm{V}_{\mathrm{D}}$ in $10 \mathrm{~Gy}$ (RBE) increments (V10-V60) extracted from the LEM-based calculated plans, and statistically significant clinical factors. To increase the power of our logistic regression model, we then limited the variables in the analysis to the $V_{D}$ in 10 Gy (RBE) increments and the ORN incidence. If the estimated odds ratio was $>1$, the variable was assumed to be a risk factor because it increased the baseline risk. SPSS ver.19 software (IBM SPSS, IBM Corporation, Somers, NY) was used for statistics.

\section{RESULTS}

\section{Maxillary ORN and Risk Factors}

The median follow-up time was of 50 months (range 8-82 months). ORN was observed in 11 patients (16.4\%) with a median time to ORN development of 24 months (range 8-54 months). Grade 1 ORN was observed in 2 patients (18.1\%), G2 in $4(36.3 \%), \mathrm{G} 3$ in $4(36.3 \%)$, and G4 ORN in 1 patient $(9.3 \%)$. In 
this latter case the patient had a microbial infection at the sphenoid bone not properly managed, and the necrosis expanded up to the right carotid channel approximately 10 months after CIRT. To avoid fatal bleeding due to carotid blow out, the patient had to perform carotid artery coiling. At the last follow up, the patient was alive without evidence of disease, and his necrosis stable. In 6 patients ORN started at the bone close to the teeth alveoli, and in one case followed the loss of one dental element. Majority of the cases had stable ORN at the last follow up (81.8\%). In 2 cases with G3 ORN a surgical sequestrotomy was performed, while in one case the defect was repaired with a temporal muscle flap. Furthermore, 3 patients in the series took advantage of hyperbaric chamber treatment to stabilize the progression of their G2 necrosis after intermittent cycles of antibiotics for recurrent infections of the necrotic area.

On univariate analysis, the tumor site, and acute mucositis $\geq$ G1 developed during CIRT were found to be statistically associated with maxillary ORN (Table 2). Majority of the patients with ORN had a tumor located in the sinonasal area (p-value 0.035). Among patients treated with CIRT who did not experience acute mucositis, none had maxillary ORN. Among those with acute mucositis, however, $22.4 \%$ also had a late effect of maxillary toxicity ( $p$-value 0.028 ). Log-rank tests for these factors confirmed that the difference between the Kaplan-Meier curves was statistically significant. In fact, tumors located in the sinonasal area had a higher probability of developing ORN over time ( $\mathrm{p}$-value 0.053 ), as for patients experiencing acute mucositis during CIRT compared to the others (p-value 0.040).

\section{Dose-Volume Risk Factors and Comparison With Published Data}

In Table 3 are reported the median cut-off value for V10-V60 and the $\mathrm{V}_{\mathrm{D}}$ correlation with the ORN incidence in our series as extracted from the LEM-based patients treatment plans. On univariate analysis, all the $\mathrm{V}_{\mathrm{D}}$ were statistically associated with maxillary ORN (V10, V20, V30, V40, V50, V55, and V60) together with the presence of teeth in the irradiated PTV. The incidence of ORN always occurred in patients where the maxillary volume received dose higher than or equal to the median cut-off. Figure 1 shows an example of one of the patients in the series that experienced ORN along with the screenshot from its treatment plans (Figure 1). Log-rank tests for all the $V_{D}$ confirmed that the survival function from ORN decreases as the irradiated maxillary volume increases, both for low and high dose levels (data not shown). Among those patients who had no upper teeth within the PTV, none reported ORN. Among those who had maxillary teeth within the PTV, 36.7\% suffered from maxillary ORN after CIRT (p-value $<0.001$ ).

Considering all the $\mathrm{V}_{\mathrm{D}}$, the tumor site, the presence of teeth within the PTV and acute mucositis, the logistic regression model identified only V60 ${ }_{\text {LEM }}$ as a significant variable in differential risk compared with baseline risk (Hazard Ratio = $1.431, \mathrm{p}=<0.001,95 \%$ CI 1,194-1,716). The risk of developing ORN increased by $43.1 \%$ if a maxillary volume $\geq 0.6 \mathrm{ml}$ was covered with at least $60 \mathrm{~Gy}(\mathrm{RBE})$ dose. When limiting the variables in the regression analysis to the $V_{D}$ in 10 Gy (RBE) increments to increase the power of prediction, the V60 LEM was still identified as independent factor significantly associated to the ORN development in our series.

When the cohort of the patients was divided into two groups according to the appearance of ORN, the average maxillary dose received by patients with ORN was higher at all volume levels, in both RBE languages (Figure 2 for both LEM and MKM treatment plans). In Figure 3 we have shown the cumulative incidence of CIRT-related ORN over time in our series when ranking the patients according to the V60 LEM.

TABLE 2 | Correlation of relevant patients clinical and tumor characteristics and ORN at univariate analysis.

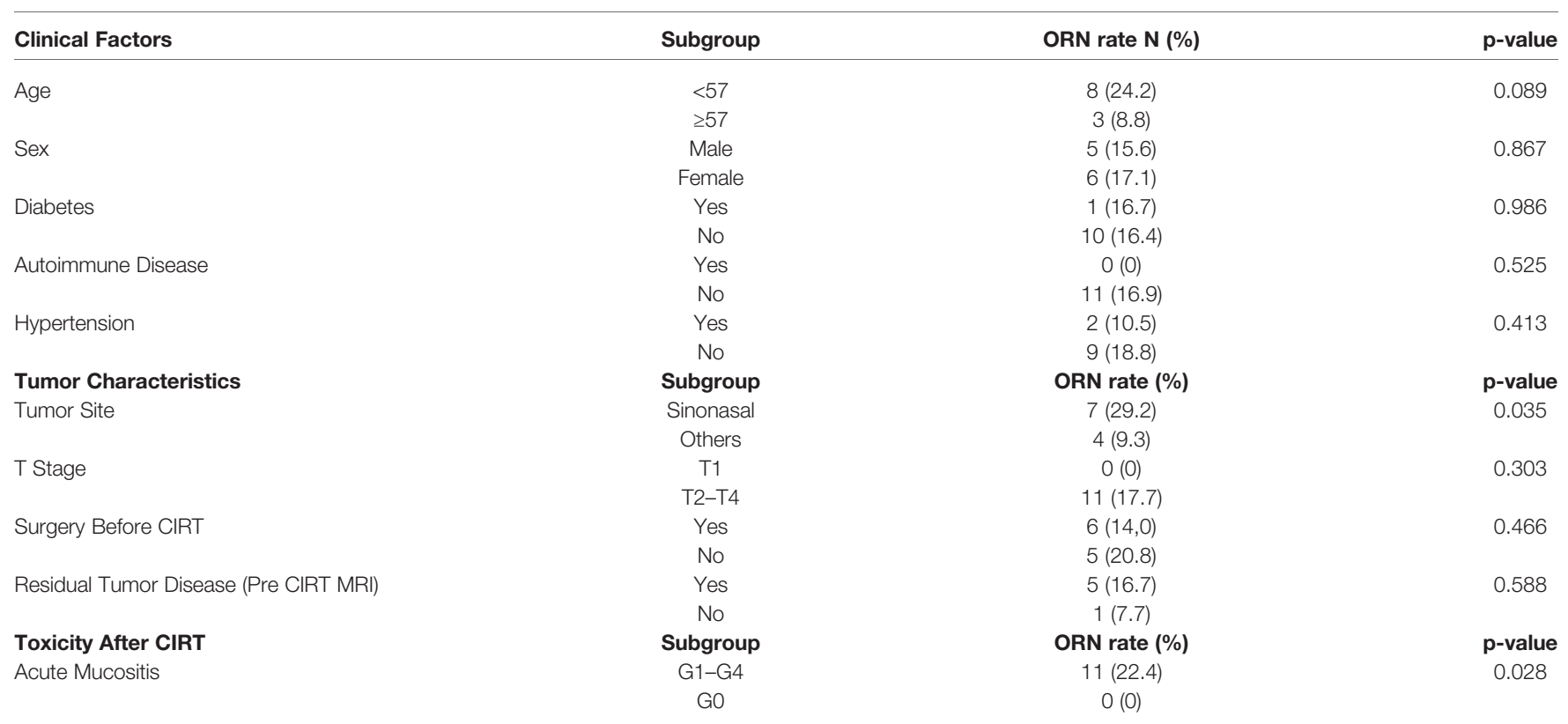


TABLE 3 | Univariate analysis of dichotomized dosimetric factors from LEMbased calculated treatment plans to assess dose-volume relationship and risk of maxillary ORN.

\begin{tabular}{lccc}
\hline V-DOSE & Subgroup & ORN (\%) & p-value \\
\hline V10 & $<8.6$ & 0 & $<0.001$ \\
V20 & $\geq 8.6$ & 32.4 & \\
& $<5.5$ & 0 & $<0.001$ \\
V30 & $\geq 5.5$ & 32.4 & \\
& $<4.4$ & 0 & $<0.001$ \\
V40 & $\geq 4.4$ & 32.4 & \\
& $<2.9$ & 0 & $<0.001$ \\
V50 & $\geq 2.9$ & 32.4 & 0.001 \\
V60 & $<1.0$ & 0 & 0.001 \\
& $\geq 1.0$ & 31.4 & \\
Teeth in PTV & $<0.6$ & 0 & $<0.001$ \\
& $\geq 0.6$ & 31.4 & \\
\hline
\end{tabular}

Teeth within the PTV were considered a risk factor for analysis.
Finally, as for comparison with previous literature dosimetric data acquired with the MKM radiobiological model (13), the LEM-based dose received by the $\mathrm{V} 50_{\mathrm{MKM}}$ of each patient in our cohort averaged over the patient population, was 55 Gy (RBE) \pm 2 SD.

\section{DISCUSSION}

Bone necrosis is an important side effect, not only when irradiating with conventional photon RT, but also after CIRT. Here we analyzed for the first time the risk factors for ORN development in patients treated with LEM-based CIRT. Although we had a small sample of patients and of ORN events, we have still shown in our series the relevance of dosimetric parameters in predicting ORN risk after CIRT, with both the biological models mostly used in CIRT facilities worldwide for treatment planning, the LEM and MKM model.
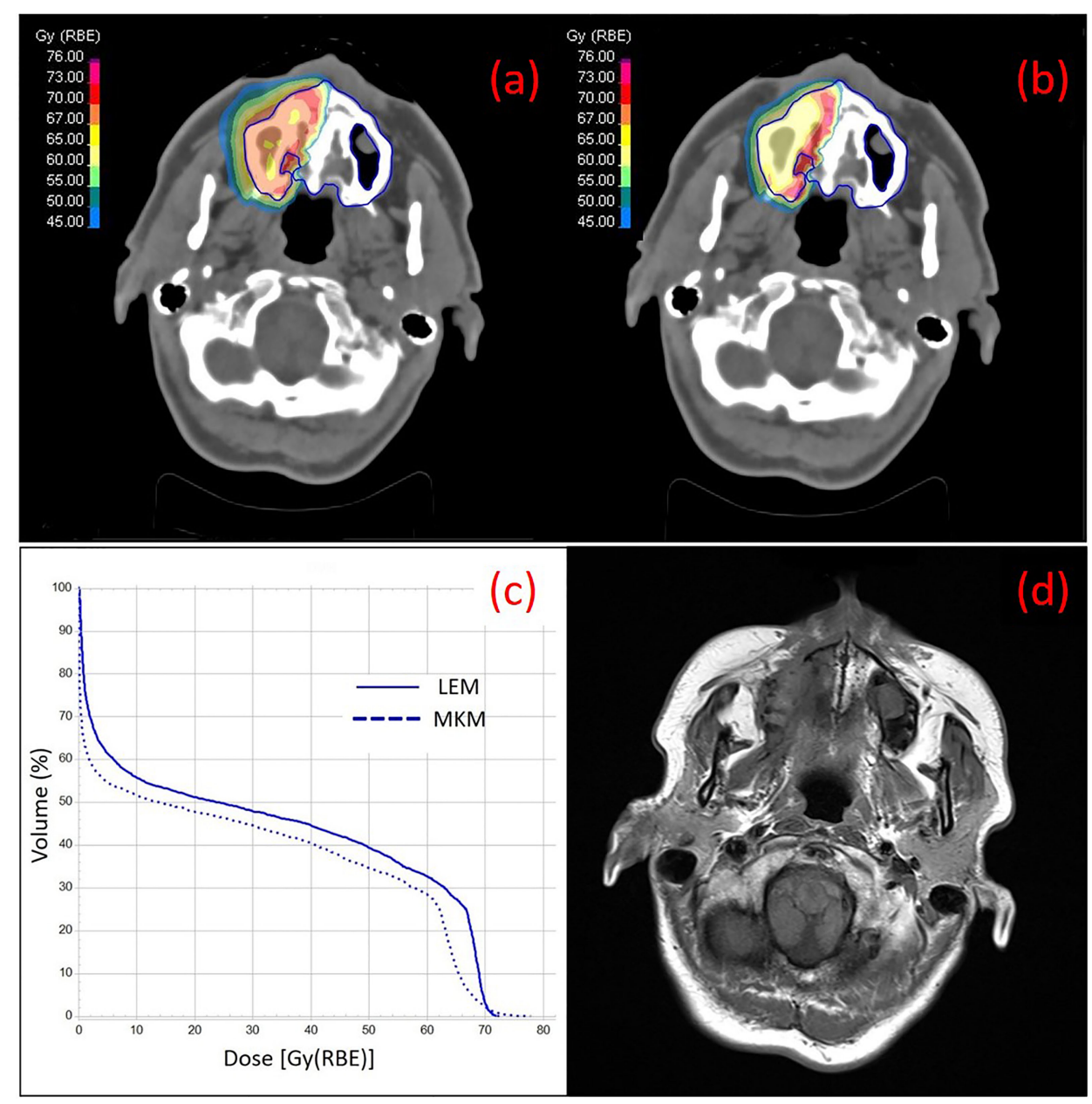

FIGURE 1 | Treatment plans on the simulation CT of one of the patients of the series with ACC at the right nasal cavity with color wash of the most representative isodoses at the maxilla (contoured in blue), calculated with LEM (A) and MKM (B) radiobiological model, are shown. The irradiation geometry consisted of 2 beam ports with couch rotation of $165^{\circ} \mathrm{C}$ and $180^{\circ} \mathrm{C}$. In (C) the DVH curves for the maxilla calculated with LEM and MKM are depicted. In (D) a T1-weighted axial image taken from the 2 years post-CIRT MRI of the same patient is depicted with typical imaging features of maxillary Grade 1 necrosis. 


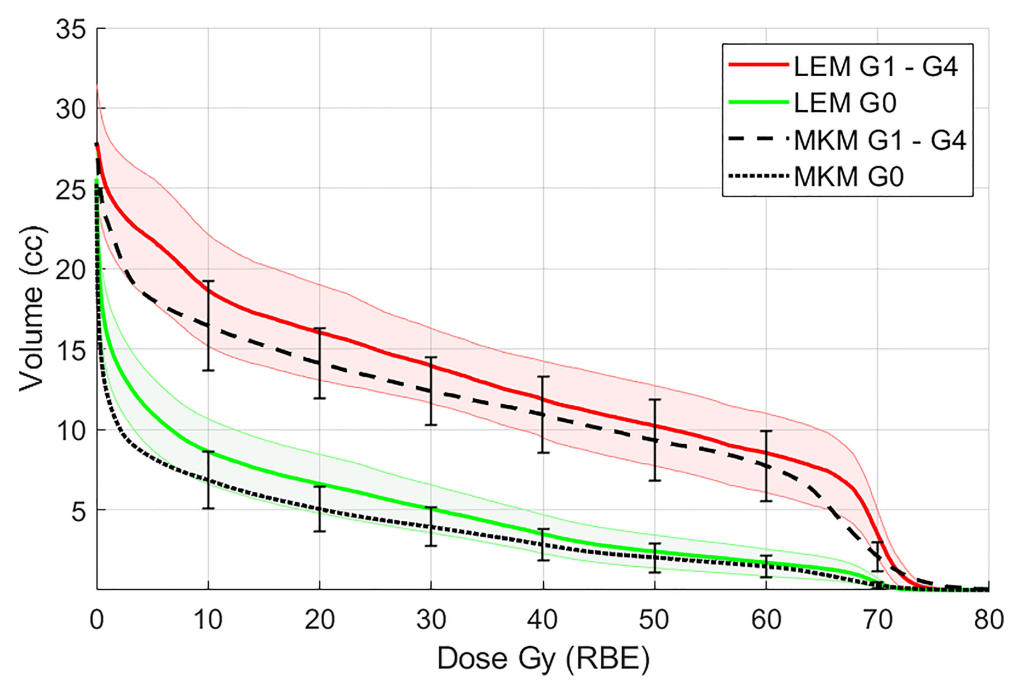

FIGURE 2 | Mean maxillary volumes at incremental dose levels from 10 to 60 Gy (RBE) calculated with the LEM model in patients with ORN (G1-G4, red solid line) vs patients without ORN (GO, green solid line). In black the maxilla DVH averaged for all the patients for the MKM model is also presented. Shaded bands and vertical error bars represent 2 SEM (Standard Error of the Mean) for the LEM and MKM model, respectively.

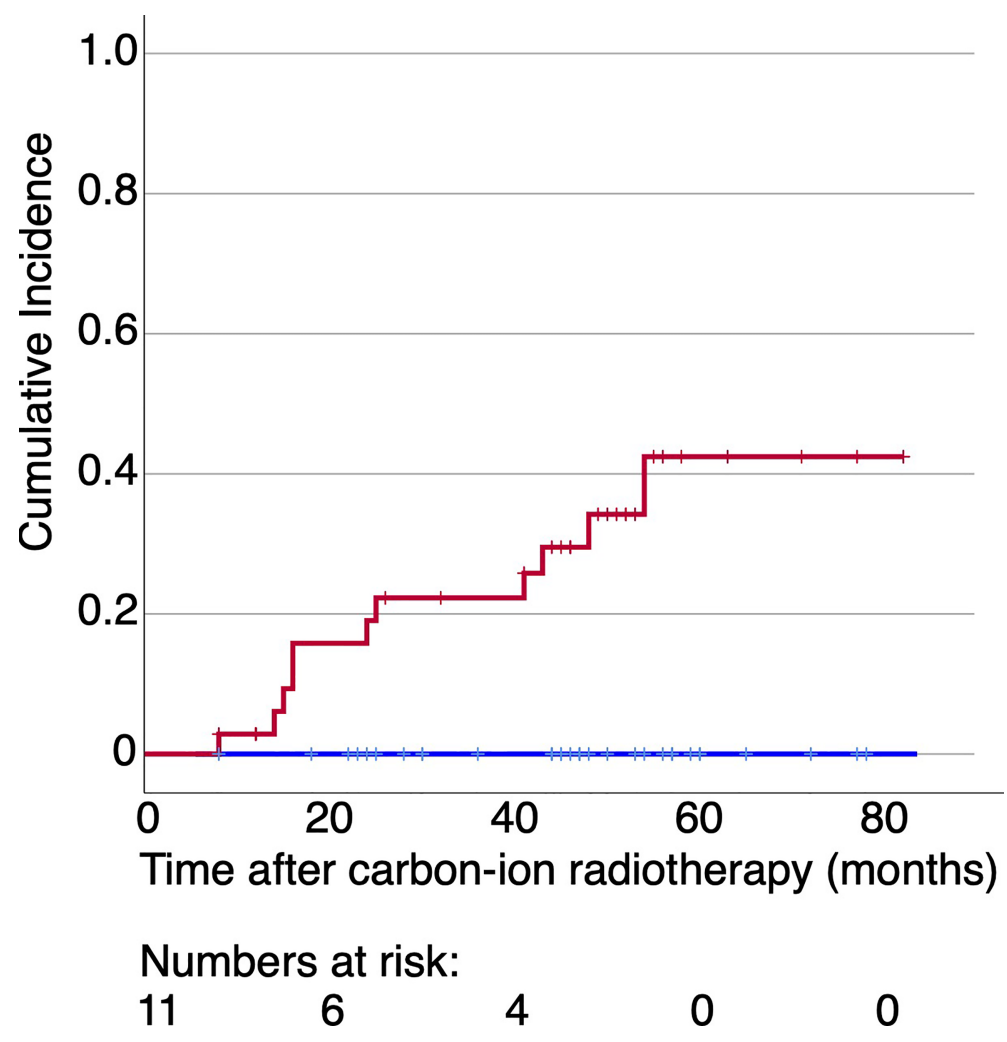

FIGURE 3 | Cumulative incidence of CIRT-related ORN over time for the patients irradiated with more than 60 Gy (RBE) at $0.6 \mathrm{ml}$ of their maxilla (red line) or not (blue line) with data extracted form LEM-based treatment plans. No patients experienced ORN in case the dose to $0.6 \mathrm{ml}$ of their maxilla was below $60 \mathrm{~Gy}$ (RBE). 
Tumor treatment, especially when curative, must not disregard ORN risk to preserve patient quality of life. However, sparing dose to the maxilla, should never be prioritized at the cost of target coverage.

With a similar study design, in Sasahara et al. the V50 was found to be a risk factor at multivariate analysis, along with the presence of teeth in the PTV, in a series with different radioresistant tumors including ACC treated with CIRT at a prescription dose of 57.6 Gy (RBE) (13). As pointed out in Musha et al., the adjustment for the RBE model is necessary in CIRT facilities outside Japan (24), when investigating for dosimetric factors predisposing to toxicity such as ORN. As part of our investigation, here we wanted to translate the V50 $0_{\mathrm{MKM}}$, significant factor for ORN reported for the Japanese series (13), into the corresponding $\mathrm{V}_{\mathrm{D}}$ extrapolated from our LEM optimized plans recalculated with the MKM Japanese radiobiological model. In our patient population, $\mathrm{V} 50_{\mathrm{MKM}}$ corresponded to V55 $5_{\mathrm{LEM}}$. The statistical significance of $55 \mathrm{~Gy}$ (RBE) was not explicitly tested, but it is in good agreement with the dose value resulting significant at our multivariate analysis

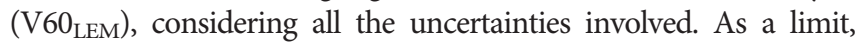
several sources of dose deviation need to be considered when comparing clinical outcomes from patient series treated with different techniques, such as the beam delivery, the different beam number per fraction and beam arrangements, physical and RBEweighted dose calculation uncertainties, the small number of patients, and different clinical characteristics (19). Furthermore, the power of the multivariate analysis presented here is low because of the small number of ORN events. Indeed, we might consider this study as a pilot trial, and for the future we aim at increasing the number of patients to produce more solid data on the clinical and dosimetric factors predisposing to ORN.

The relevance of the high doses when irradiating with CIRT in comparison to photon RT was pointed out in Ikawa et al., where 76 patients with oral nonsquamous cell carcinoma treated with CIRT at mostly with $64 \mathrm{~Gy}(\mathrm{RBE})$ prescription dose in 16 fractions equivalent to $89.6 \mathrm{~Gy}(\mathrm{RBE})$ at a fractionation of $2 \mathrm{~Gy}$ (RBE) per fraction, experienced $11.8 \%$ G3 ORN requiring surgery (25). Furthermore, in Naganawa et al. reporting on CIRT in 19 patients with oral malignant mucosal melanoma treated with $57.6 \mathrm{~Gy}$ (RBE) prescription dose in 16 fractions, all Grade 2 and 3 ORN were derived from the alveolar bone in the high dose-irradiated volume. Despite this, the mean $\mathrm{V}^{5} 0_{\mathrm{MKM}}$ of the maxilla or mandible was $12.5 \mathrm{ml}$ (range, 0-23.4 ml) for patients with Grade $0-1$, and $12.5 \mathrm{ml}$ (range, 3.1-22.3 ml) for patients with Grade 2-3 ORN, respectively, with a difference not statistically significant (26).

The impact of lower doses after CIRT on the mandible ORN onset has been recently reported to be significant when irradiating tumors localized at the oral cavity and oropharynx, for the $\mathrm{V} 30_{\mathrm{MKM}^{-}}-\mathrm{V} 45_{\mathrm{MKM}}$ at the mandible, and in case of ${\mathrm{V} 10_{\mathrm{MKM}^{-}}}^{-}$ V30 $0_{M K M}$ to the teeth (24). In our study, all the $\mathrm{V}_{\mathrm{D}}\left(\mathrm{V} 10_{\mathrm{LEM}^{-}} \mathrm{V} 60\right.$ LEM) tested at univariate analysis were statistically correlated with the occurrence of ORN. Maxillary ORN cases occurred in the subgroup of patients with $\mathrm{V}_{\mathrm{D}}$ larger than- or equal- to the median volume for that DVH as it has been reported also in Sasahara et al. although with a different biological model (13). This indicates that the risk of ORN in our analysis of LEM-based calculated treatment plans might be enhanced both for larger bone volumes exposed to a low dose bath $(<50 \mathrm{~Gy}(\mathrm{RBE}))$, and for smaller bone volumes exposed to a high dose $>55 \mathrm{~Gy}$ (RBE). This finding might be translated into the importance of maximizing the dose conformity at the tumor target while sparing sensitive organs for toxicity, such as the maxilla, minimizing the volume receiving high doses to the maxilla in the range $\mathrm{V} 55_{\mathrm{LEM}}-\mathrm{V} 60_{\mathrm{LEM}}$, and the dose bath at lower doses. In this regard, CIRT in general for its physical and biological peculiarities, and the spot scanning beam delivery technique, might represent an advantage as postulated in Ikawa et al. when treating radioresistant tumors that requires high doses (25). In our series, even if the latency time for the ORN onset was similar as in Sasahara et al. (13), ORN incidence was less probable, not only because of the shorter follow up time in our series ( 50 vs 79 months), but possibly for the active spot scanning technique (vs passive beam) routinely employed at CNAO for treatments.

Not surprisingly, in our study with ACC in different locations in the maxillofacial area, ORN was mainly found after CIRT at the sinonasal site. In this regard, in Koto et al., over the 458 patients with sinonasal malignancies treated with different CIRT prescription doses but more frequently with 64 Gy (RBE) in 16 fractions, maxillary ORN was the most frequent late toxicity together with visual impairment, with G3 ORN reported in 18 patients (4\%) and grade 2 in $36(8 \%)$ (27).

In our series, the presence of superior dental elements within the PTV correlated with the onset and development of maxillary ORN at univariate analysis. In Bhattacharyya et al. the number of teeth irradiated with more than 50 Gy (RBE) was a significant independent risk factor for the development of oronasal fistula, which is also a late complication of CIRT. In order to reduce the risk, the authors suggested minimizing the number of teeth at a maximum of 2 within the volume irradiated with more than 50 Gy (RBE) (20). These data, together with data with conventional photon RT on the impact of dental infection on ORN incidence (28), confirm the benefit of pre-CIRT prophylactic dental avulsion and oral hygiene control before CIRT and during follow up adopted in CIRT facilities around the world, and at our center. Acute mucositis developed during the CIRT course (also significantly correlated with ORN in our series) together with the high radiation dose to the maxilla alveolar bone portion, might lead to superficial and then deep periodontal disease, gingival recession with increase susceptibility to infection with progressive bone injury leading to ORN (24). The elimination of odontostomatological risk factors (traumatic, inflammatory, and infectious events) and not, before the start of CIRT and during follow up, is of considerable importance for the outcome of the treatments and the quality of life of the patients. Time management of teeth extractions should also be strictly observed both before and after CIRT, and dental extraction protocol should be based on the individual plan isodose maps in case is not avoidable. As previously reported, teeth extraction or surgical procedure whenever not amenable should be performed not earlier than 1 year after CIRT to avoid ORN 
progression to soft tissue and skin, and more serious sequelae with related deterioration of quality of life (29). According to the median time of onset of maxillary ORN in our analysis, it would be advisable to wait at least for 24 months after CIRT before any bone intervention. Conservative measures are recommended for early stage ORN, reserving surgery at later stages when no other medical options are available.

In our series, we investigated also pre-CIRT surgery as risk factor for ORN. According to Marunick et al., pre-RT surgical risk involves tissue trauma, reduced blood supply, cell death, collagen lysis, and greater metabolic challenge to repair. Surprisingly, here postoperative CIRT was not correlated with increased ORN risk differently from Marunick et al., where only the 4 patients included in the study who had jointly received both definitive tumor resection and neutron RT developed ORN at the residual maxillary orbital complex (30).

In summary, among all the toxicities of patients treated with CIRT in the head and neck area, ORN is one of the most serious and debilitating chronic post-treatment sequelae. Here, we have pointed out the $\mathrm{V} 55_{\mathrm{LEM}^{-}}-\mathrm{V} 60_{\mathrm{LEM}}$ irradiated volumes as most important factor correlated with ORN. The limitations of our study include the small patient number and the short follow up. For the future, clinical trials with larger patients' number and longer follow up are warranted. Based on our experience enriched by literature data, we might further summarize the recommendations for the optimal strategies to reduce ORN risk onset when irradiating tumors at the skull base, especially in facilities using the LEM-based CIRT: 1) to perform dental examination and prophylactic extraction procedures before CIRT if necessary, and close dentist follow up for all the 2-year period after CIRT more at risk of developing ORN, especially in case of patients with the maxilla covered for large volume by dose higher than $55 \mathrm{~Gy}(\mathrm{RBE}) ; 2$ ) to maximize the dose conformity to the maxilla by reducing volumes irradiated with dose higher than

\section{REFERENCES}

1. Marx RE. Osteoradionecrosis: A New Concept of its Pathophysiology. J Oral Maxillofac Surg (1983) 41(5):283-8. doi: 10.1016/0278-2391(83)90294-X

2. Leonetti JP, Weishaar JR, Gannon D, Harmon GA, Block A, Anderson DE. Osteoradionecrosis of the Skull Base. J Neurooncol (2020) 150(3):477-82. doi: $10.1007 /$ s11060-020-03462-3

3. Tsai CJ, Hofstede TM, Sturgis EM, Garden AS, Lindberg ME, Wei Q, et al. Osteoradionecrosis and Radiation Dose to the Mandible in Patients With Oropharyngeal Cancer. Int J Radiat Oncol Biol Phys (2013) 85(2):415-20. doi: 10.1016/j.ijrobp.2012.05.032

4. Wahl MJ. Osteoradionecrosis Prevention Myths. Int J Radiat Oncol Biol Phys (2006) 64(3):661-9. doi: 10.1016/j.ijrobp.2005.10.021

5. Lee AW, Ng WT, Chan LL, Hung WM, Chan CC, Sze HC, et al. Evolution of Treatment for Nasopharyngeal Cancer-Success and Setback in the IntensityModulated Radiotherapy Era. Radiother Oncol (2014) 110(3):377-84. doi: 10.1016/j.radonc.2014.02.003

6. Tinganelli W, Durante M. Carbon Ion Radiobiology. Cancers (Basel) (2020) 12(10):3022. doi: 10.3390/cancers 12103022

7. Orlandi E, Iacovelli NA, Bonora M, Cavallo A, Fossati P. Salivary Gland. Photon Beam and Particle Radiotherapy: Present and Future. Oral Oncol (2016) 60:146-56. doi: 10.1016/j.oraloncology.2016.06.019

8. Sulaiman NS, Demizu Y, Koto M, Saitoh JI, Suefuji H, Tsuji H, et al. Multicenter Study of Carbon-Ion Radiation Therapy for Adenoid Cystic Carcinoma of the Head and Neck: Subanalysis of the Japan Carbon-Ion
55 Gy (RBE) and the dose bath below 50 Gy (RBE); 3) to avoid teeth in the PTV especially if the tumor does not involve the maxilla, and in case it is inevitable, to include not more than 2 teeth in the high dose PTV; 4) to prefer conservative measures and avoid surgical traumatic procedures after ORN onset. In case of unamenable necrosectomy after CIRT, our recommendation might be to remove the bone around the necrotic tissue by including at least the V55 LEM isodose and higher, in order to facilitate tissue post-surgical healing.

\section{DATA AVAILABILITY STATEMENT}

The raw data supporting the conclusions of this article will be made available by the authors, without undue reservation.

\section{ETHICS STATEMENT}

The studies involving human participants were reviewed and approved by the Ethical committee San Matteo Hospital, Pavia (Italy). The patients/participants provided their written informed consent to participate in this study.

\section{AUTHOR CONTRIBUTIONS}

Conceptualization: BV, SR, and EO. Methodology and statistics: BV, SR, SM, EO, MM, and SR. Writing: MM and BV. Review and Editing: BV, SR, MM, MB, SRO, RI, AMC, SI, LP, MC, SM, and EO. Supervision: BV and EO. All authors listed have made a substantial, direct, and intellectual contribution to the work and approved it for publication.

Radiation Oncology Study Group (J-CROS) Study (1402 Hn). Int J Radiat Oncol Biol Phys (2018) 100(3):639-46. doi: 10.1016/j.ijrobp.2017.11.010

9. Akbaba S, Bostel T, Lang K, Bahadir S, Lipman D, Schmidberger H, et al. Large German Multicenter Experience on the Treatment Outcome of 207 Patients With Adenoid Cystic Carcinoma of the Major Salivary Glands. Front Oncol (2020) 10:593379. doi: 10.3389/fonc.2020.593379

10. Ronchi S, Vischioni B, Bonora M, Barcellini A, Locati LD, Castelnuovo P, et al. Managing Locally Advanced Adenoid Cystic Carcinoma of the Head and Neck During the COVID-19 Pandemic Crisis: Is This the Right Time for Particle Therapy? Oral Oncol (2020) 106:104803. doi: 10.1016/j.oraloncology.2020.104803

11. Vischioni B, Dhanireddy B, Severo C, Bonora M, Ronchi S, Vitolo V, et al. Reirradiation of Salivary Gland Tumors With Carbon Ion Radiotherapy at CNAO. Radiother Oncol (2020) 145:172-7. doi: 10.1016/j.radonc.2020.01.004

12. Loap $\mathrm{P}$, Vischioni $\mathrm{B}$, Bonora $\mathrm{M}$, Ingargiola $\mathrm{R}$, Ronchi $\mathrm{S}$, Vitolo $\mathrm{V}$, et al. Biological Rationale and Clinical Evidence of Carbon Ion Radiation Therapy for Adenoid Cystic Carcinoma: A Narrative Review. Front Oncol (2021) 11:789079. doi: 10.3389/fonc.2021.789079

13. Sasahara G, Koto M, Ikawa H, Hasegawa A, Takagi R, Okamoto Y, et al. Effects of the Dose-Volume Relationship on and Risk Factors for Maxillary Osteoradionecrosis After Carbon Ion Radiotherapy. Radiat Oncol (2014) 9 (1):92. doi: 10.1186/1748-717X-9-92

14. Kanai T, Endo M, Minohara S, Miyahara N, Koyama-ito H, Tomura H, et al. Biophysical Characteristics of HIMAC Clinical Irradiation System for HeavyIon Radiation Therapy. Int J Radiat Oncol Biol Phys (1999) 44(1):201-10. doi: 10.1016/s0360-3016(98)00544-6 
15. Inaniwa T, Furukawa T, Kase Y, Matsufuji N, Toshito T, Matsumoto Y, et al. Treatment Planning for a Scanned Carbon Beam With a Modified Microdosimetric Kinetic Model. Phys Med Biol (2010) 55(22):6721-37. doi: 10.1088/0031-9155/55/22/008

16. Krämer M, Jäkel O, Haberer T, Kraft G, Schardt D, Weber U. Treatment Planning for Heavy-Ion Radiotherapy: Physical Beam Model and Dose Optimization. Phys Med Biol (2000) 45(11):3299-317. doi: 10.1088/00319155/45/11/313

17. Molinelli S, Magro G, Mairani A, Matsufuji N, Kanematsu N, Inaniwa T, et al. Dose Prescription in Carbon Ion Radiotherapy: How to Compare Two Different RBE-Weighted Dose Calculation Systems. Radiother Oncol (2016) 120(2):307-12. doi: 10.1016/j.radonc

18. Facoetti A, Vischioni B, Ciocca M, Ferrarini M, Furusawa Y, Mairani A, et al. In Vivo Radiobiological Assessment of the New Clinical Carbon Ion Beams at CNAO. Radiat Prot Dosimet (2015) 166(1-4):379-82. doi: 10.1093/rpd/ ncv145

19. Molinelli S, Bonora M, Magro G, Casale S, Dale JE, Fossati P, et al. RBEWeighted Dose in Carbon Ion Therapy for ACC Patients: Impact of the RBE Model Translation on Treatment Outcomes. Radiother Oncol (2019) 141:22733. doi: 10.1016/j.radonc.2019.08.022

20. Bhattacharyya T, Koto M, Ikawa H, Hayashi K, Hagiwara Y, Tsuji H. Assessment of Risk Factors Associated With Development of Oronasal Fistula as a Late Complication After Carbon-Ion Radiotherapy for Head and Neck Cancer. Radiother Oncol (2020) 144:53-8. doi: 10.1016/ j.radonc.2019.10.015

21. Bonora M, Vischioni B, Caivano D, Hasegawa A, Vitolo V, Ronchi S. EP-1189 Adenoid Cystic Carcinoma Of The Head And Neck Treated With Carbon Ion Radiotherapy At CNAO. Radiother Oncol (2019) 133:S659. doi: 10.1016/ S0167-8140(19)31609-3

22. Common Terminology Criteria for Adverse Events (CTCAE) Protocol Development CTEP. Available at: https://ctep.cancer.gov/protocolDevelopment/electronic_ applications/ctc.htm (Accessed on 15 July 2021).

23. Tuan J, Vischioni B, Fossati P, Srivastava A, Vitolo V, Iannalfi A, et al. Initial Clinical Experience With Scanned Proton Beams at the Italian National Center for Hadrontherapy (CNAO). J Radiat Res (2013) 54 Suppl 1(Suppl 1):i31-42. doi: 10.1093/jrr/rrt036

24. Musha A, Shimada H, Kubo N, Kawamura H, Okano N, Sato H, et al. Clinical Features and Dosimetric Evaluation of Carbon Ion Radiation-Induced Osteoradionecrosis of Mandible in Head and Neck Tumors. Radiother Oncol (2021) 161:205-10. doi: 10.1016/j.radonc.2021.06.022
25. Ikawa H, Koto M, Hayashi K, Tonogi M, Takagi R, Nomura T, et al. Feasibility of Carbon-Ion Radiotherapy for Oral non-Squamous Cell Carcinomas. Head Neck (2019) 41(6):1795-803. doi: 10.1002/hed.25618

26. Naganawa K, Koto M, Takagi R, Hasegawa A, Ikawa H, Shimozato K, et al. Organizing Committee for the Working Group for Head-And-Neck Cancer. Long-Term Outcomes After Carbon-Ion Radiotherapy for Oral Mucosal Malignant Melanoma. J Radiat Res (2017) 58(4):517-22. doi: 10.1093/jrr/ rrw117

27. Koto M, Demizu Y, Saitoh JI, Suefuji H, Tsuji H, Okimoto T, et al. Definitive Carbon-Ion Radiation Therapy for Locally Advanced Sinonasal Malignant Tumors: Subgroup Analysis of a Multicenter Study by the Japan Carbon-Ion Radiation Oncology Study Group (J-CROS). Int J Radiat Oncol Biol Phys (2018) 102(2):353-61. doi: 10.1016/j.ijrobp.2018.05.074

28. Wang TH, Liu CJ, Chao TF, Chen TJ, Hu YW. Risk Factors for and the Role of Dental Extractions in Osteoradionecrosis of the Jaws: A National-Based Cohort Study. Head Neck (2017) 39(7):1313-21. doi: 10.1002/hed.24761

29. Dell'Era V, Aluffi Valletti P, Garzaro G, Garzaro M. Maxillo-Mandibular Osteoradionecrosis Following C-Ion Radiotherapy: Clinical Notes and Review of Literature. Eur J Inflammation (2020) 18:1-6. doi: 10.1177/2058739220934562

30. Marunick MT, Bahu SJ, Aref A. Osteoradionecrosis of the Maxillary-Orbital Complex After Neutron Beam Radiotherapy. Otolaryngol Head Neck Surg (2000) 123(3):224-8. doi: 10.1067/mhn.2000.107403

Conflict of Interest: The authors declare that the research was conducted in the absence of any commercial or financial relationships that could be construed as a potential conflict of interest.

Publisher's Note: All claims expressed in this article are solely those of the authors and do not necessarily represent those of their affiliated organizations, or those of the publisher, the editors and the reviewers. Any product that may be evaluated in this article, or claim that may be made by its manufacturer, is not guaranteed or endorsed by the publisher.

Copyright (C) 2022 Vischioni, Russo, Meuli, Bonora, Ronchi, Ingargiola, Camarda Imparato, Preda, Ciocca, Molinelli and Orlandi. This is an open-access article distributed under the terms of the Creative Commons Attribution License (CC BY). The use, distribution or reproduction in other forums is permitted, provided the original author(s) and the copyright owner(s) are credited and that the original publication in this journal is cited, in accordance with accepted academic practice. No use, distribution or reproduction is permitted which does not comply with these terms. 